\title{
Dynamic Boundary Control of the Timoshenko Beam*
}

\author{
ÖMER MORGÜL†
}

Key Words-Distributed parameter systems; partial differential equations; boundary-value problems; stability; Lyapunov methods.

\begin{abstract}
We consider a clamped-free Timoshenko beam To stabilize the beam vibrations, we propose a dynamic boundary control law applied at the free end of the beam. We prove that with the proposed control law, the beam vibrations uniformly and exponentially decay to zero. The proof uses a Lyapunov functional based on the energy of the system.
\end{abstract}

\section{Introduction}

IN THIS PAPER, we study the uniform stabilization of the clamped-free Timoshenko beam with dynamic boundary control. The Timoshenko beam model is a linear beam model which accounts for both the rotatory inertia of the beam cross-sections and the deffection due to shear effects. This model is a more accurate one than both the Euler-Bernoulli beam model which yields good results when the cross-sectional dimensions of the beam are small in comparison with the length of the beam, and the Rayleigh beam model in which only the rotatory inertia of the beam cross-sections are taken into account, see Meirovitch (1967). Assuming that the beam is homogeneous with uniform cross-sections, the equations of motion of the Timoshenko beam is described by the following set of equations: for $x \in(0, L)$

$$
\begin{gathered}
\rho \frac{\partial^{2} u}{\partial t^{2}}-K\left(\frac{\partial^{2} u}{\partial x^{2}}-\frac{\partial \phi}{\partial x}\right)=0, \\
I_{\rho} \frac{\partial^{2} \phi}{\partial t^{2}}-E I \frac{\partial^{2} \phi}{\partial x^{2}}+K\left(\phi-\frac{\partial u}{\partial x}\right)=0,
\end{gathered}
$$

where $L$ is the length of the beam, $t$ is the time variable, $x$ is the space coordinate along the beam in its equilibrium position, $u(x, t)$ is the deflection of the beam from its equilibrium position, which is characterized by $u=0, \phi(x, t)$ is the angle of rotation of the beam cross-sections due to bending, for more precise definitions, see Meirovitch (1967). The coefficients $\rho, I_{\rho}$ and $E I$ are the mass per unit length, the mass moment of inertia of the beam cross-sections and the flexural rigidity of the beam, respectively. The coefficient $K$ is equal to $k G A$ where $G$ is the shear modulus, $A$ is the cross-sectional area and $k$ is a numerical factor depending on the shape of the beam cross-sections. All coefficients are assumed to be constant.

Equations (1) and (2) can be obtained through Hamilton's principle by using the natural energy of the beam given by:

$$
E_{\mathrm{B}}(t)=\frac{1}{2} \int_{0}^{L}\left\{\rho u_{t}^{2}+I_{\rho} \phi_{t}^{2}+K\left(\phi-u_{x}\right)^{2}+E I \phi_{x}^{2}\right\} \mathrm{d} x,
$$

* Received 7 May 1991; revised 24 December 1991; received in final form 1 May 1992. The original version of this paper was not presented at any IFAC meeting. This paper was recommended for publication in revised form by Associate Editor T. Başar under the direction of Editor $\mathrm{H}$. Kwakernaak.

† Department of Electrical and Electronics Engineering, at Bilkent University, Ankara, Turkey. where a subscript letter denotes the partial differential with respect to the corresponding letter, e.g. $u_{t}=\partial u / \partial t$. In (3), the first two terms in the integral represent the kinetic energy due to translation and rotation and the last two terms represent the potential energy due to shearing deformation and bending, respectively, see Meirovich (1967).

The boundary conditions we have are: $t \geq 0$

$$
\begin{gathered}
u(0, t)=0, \quad \phi(0, t)=0, \\
K\left(\phi(L, t)-u_{x}(L, t)\right)=f_{1}(t), \quad-E I \phi_{x}(L, t)=f_{2}(t),
\end{gathered}
$$

where (4) is the boundary conditions at the clamped end, (5) gives the boundary conditions at the free end, $f_{1}(t)$ and $f_{2}(t)$ are the boundary control force and the boundary control moment.

Our aim in this paper is to find appropriate control laws for $f_{1}(t)$ and $f_{2}(t)$ so that the energy $E_{\mathbf{B}}(t)$ given by (3) asymptotically and uniformly decays to zero.

In recent years, the boundary control of systems described by partial differential equations has become an important research area. Chen (1979) established the uniform stabilization of the wave equation in any space dimension with the boundary control. Recently, Chen et al. (1987) established the uniform stabilization of the Euler-Bernoulli beam and Kim and Renardy (1987) obtained similar results for the Timoshenko beam. Recently these results have been extended to the rotating flexible structures, see Morgül $(1990,1991 \mathrm{a}, \mathrm{b})$. A good source of references to papers in which the boundary control techniques are treated can be found in Lagnese (1989). The stabilizing control laws presented here are more general than that of Kim and Renardy (1987), hence our results could be interpreted as a generalization of the results presented therein.

This paper is organized as follows. In Section 2, we propose a stabilizing control law and show that the system under investigation is well-posed, i.e. there exists a solution and this solution is unique in an appropriate space. In the Section 3 we show that the solutions decay exponentially fast to zero. In the Section 4, we present some numerical results, and finally we give some concluding remarks.

\section{Existence and uniqueness of the solutions}

To stabilize the system given by (1), (2), (4)-(5), we propose the following feedback control laws: for $i=1,2$

$$
\dot{w}_{i}=A_{i} w_{i}+b_{i} r_{i}(t), \quad f_{i}(t)=c_{i}^{T} w_{i}+d_{i} r_{i}(t),
$$

where, for $i=1,2, w_{i} \in \mathbf{R}^{n_{i}}$ is the actuator state, $A_{i} \in \mathbf{R}^{n_{i} \times n_{i}}$ is a constant matrix, $b_{i}, c_{i} \in \mathbf{R}^{n_{i}}$ are constant column vectors, the superscript $T$ stands for transpose, $d_{i} \in \mathbf{R}$ is a constant real number and $r_{i}(t)$ is defined as:

$$
r_{1}(t)=u_{t}(L, t), \quad r_{2}(t)=\phi_{t}(L, t), \quad t \in \mathbf{R} .
$$

We note that for $i=1(i=2$, respectively) (7) and (8) give the equations for the actuator whose input is $u_{t}(L, t)$ $\left(\phi_{t}(L, t)\right.$, respectively) and the output is the boundary control force $f_{1}(t)$ (the boundary control torque $f_{2}(t)$, respectively) 
We assume the following throughout this work: Assumptions. For $i=1,2$.

(1) All eigenvalues of the matrix $A_{i}$ are in the open left half of the complex plane,

(2) the triplet $\left(A_{i}, b_{i}, c_{i}\right)$ is both observable and controllable, (3) $d_{i}>0$; furthermore for some $\gamma_{i}>0$, such that $d_{i}>\gamma_{i}$, we have the following:

$$
\mathscr{R} e\left\{h_{i}(j \omega)\right\}>\gamma_{i}, \quad i=1,2, \omega \in \mathbf{R},
$$

where $\mathscr{P}_{e}$ denotes the real part of a complex number and for $i=1,2, h_{i}(s)=d_{i}+c_{i}^{r}\left(s I-A_{i}\right)^{-1} b_{i}$ is the actuator transfer function.

Assumption 3 implies that, for $i=1,2$, the actuator transfer function $h_{i}(s)$ is a strictly positive real function. Let the Assumptions 1-3 stated above hold. Then, it follows from the Kalman-Yacubovitch lemma that, for $i=1,2$, given any symmetric positive definite matrix $Q_{i} \in \mathbf{R}^{n_{i} \times n_{i}}$, there exist a symmetric positive definite matrix $P_{i} \in \mathbf{R}^{n_{i} \times n_{i}}$ and a vector $q_{i} \in \mathbf{R}^{n_{i}}$ satisfying:

$A_{i}^{T} P_{i}+P_{i} A_{i}=-q_{i} q_{i}^{T}-\varepsilon_{i} Q_{i}, \quad P_{i} b_{i}-\frac{1}{2} c_{i}=\sqrt{\left(d_{i}-\gamma_{i}\right)} q_{i}$,

provided that $\varepsilon_{i}>0$ is sufficiently small, see Vidyasagar (1978).

To analyze the system given by (1), (2), (4)-(7), we define the function space $\mathscr{H}$ as follows:

$$
\begin{aligned}
\mathscr{H}:=\left\{\left(u_{1} u_{2} \phi_{1} \phi_{2} x_{1} x_{2}\right)^{T}\right) \mid u_{1} \in \mathbf{H}_{0}^{1}, \\
\\
\left.u_{2} \in \mathbf{L}^{2}, \phi_{1} \in \mathbf{H}_{0}^{1}, \phi_{2} \in \mathbf{L}^{2}, x_{1} \in \mathbf{R}^{n_{1}}, x_{2} \in \mathbf{R}^{n_{2}}\right\},
\end{aligned}
$$

where the spaces $\mathbf{L}^{2}$ and $\mathbf{H}_{0}^{k}$ are defined as follows:

$$
\begin{gathered}
\mathbf{L}^{2}=\left\{f:[0, L] \rightarrow \mathbf{R} \mid \int_{0}^{L} f^{2} \mathrm{~d} x<\infty\right\}, \\
\mathbf{H}_{0}^{k}=\left\{f \in \mathbf{L}^{2} \mid f, f^{\prime}, f^{\prime \prime}, \ldots, f^{(k)} \in \mathbf{L}^{2}, \quad f(0)=0\right\} .
\end{gathered}
$$

The equations (1), (2), (4)-(8) can be written in the following abstract form:

$$
\dot{z}=A z, \quad z(0) \in \mathscr{H},
$$

where $z=\left(u u, \phi \phi, w_{1} w_{2}\right)^{T} \in \mathscr{H}$, the operator $A: \mathscr{H} \rightarrow \mathscr{H}$ is a linear unbounded operator defined as

$$
A y=\left[\begin{array}{c}
\frac{K}{\rho} \frac{\partial^{2} u_{1}}{\partial x^{2}}-\frac{K}{\rho} \frac{\partial \phi_{1}}{\partial x} \\
\phi_{2} \\
\frac{E I}{I_{\rho}} \frac{\partial^{2} \phi_{1}}{\partial x^{2}}+\frac{K}{I_{p}} \phi_{1}-\frac{K}{I_{\rho}} \frac{\partial u_{1}}{\partial x} \\
A_{1} x_{1}+b_{1} u_{2}(L) \\
A_{2} x_{2}+b_{2} \phi_{2}(L)
\end{array}\right]
$$

where $y=\left(u_{1} u_{2} \phi_{1} \phi_{2} x_{1} x_{2}\right)^{T}$. The domain $D(A)$ of the operator $A$ is defined as:

$$
\begin{gathered}
D(A):=\left\{\left(u_{1} u_{2} \phi_{1} \phi_{2} x_{1} x_{2}\right)^{T} \mid u_{1} \in \mathbf{H}_{0}^{2}, u_{2} \in \mathbf{H}_{0}^{1}, \phi_{1} \in \mathbf{H}_{0}^{2},\right. \\
\phi_{2} \in \mathbf{H}_{0}^{1}, x_{1} \in \mathbf{R}^{n_{1}}, x_{2} \in \mathbf{R}^{n_{2}} \\
K\left(\frac{\partial u_{1}}{\partial x}(L)-\phi_{1}(L)\right)+c_{1}^{T} x_{1}+d_{1} u_{2}(L)=0, \\
\left.E I \frac{\partial \phi_{1}}{\partial x}(L)+c_{2}^{T} x_{2}+d_{2} \phi_{2}(L)=0\right\} .
\end{gathered}
$$

It can easily be shown that $D(A)$ is dense in $\mathscr{H}$. We note that a classical solution of $(13)$ is defined as an $\mathscr{H}$-valued function $z(t)$, which is continuous for $t \geq 0$, continuously differentiable and $z(t) \in D(A)$ for $t>0$, and (13) is satisfied.

Let the Assumptions 1-3 hold, let, for $i=1,2, Q_{i} \in \mathbf{R}^{n_{i} \times n_{i}}$ be an arbitrary symmetric positive definite matrix and let $P_{i} \in \mathbf{R}^{n_{i} \times n_{i}}, q_{i} \in \mathbf{R}^{n_{i}}$ be the solutions of (9) where $P_{i}$ is also a symmetric and positive definite matrix. In $\mathscr{H}$, we define the following inner-product:

$$
\begin{aligned}
\langle y, \tilde{y}\rangle_{\mathscr{H}}=\int_{0}^{L} & \left\{K \frac{\partial u_{1}}{\partial x} \frac{\partial \tilde{u}_{1}}{\partial x}\right. \\
+ & \left.\rho u_{2} \tilde{u}_{2}+E I \frac{\partial \phi_{1}}{\partial x} \frac{\partial \tilde{\phi}_{1}}{\partial x}+I_{\rho} \phi_{2} \bar{\phi}_{2}\right\} \mathrm{d} x \\
& \quad+x_{1}^{T} P_{1} \tilde{x}_{1}+\bar{x}_{1}^{T} P_{1} x_{1}+x_{2}^{T} P_{2} \tilde{x}_{2}+\tilde{x}_{2}^{T} P_{2} x_{2}
\end{aligned}
$$

where $\tilde{y}=\left(\begin{array}{llllll}\bar{u}_{1} & \tilde{u}_{2} & \tilde{\phi}_{1} & \tilde{\phi}_{2} & \tilde{x}_{1} & \tilde{x}_{2}\end{array}\right)^{T} \in \mathscr{H}$. It can easily be shown that $\mathscr{H}$, together with the inner product defined by (16), becomes a Hilbert space.

In the sequel, we need the following inequalities:

$$
\begin{gathered}
u^{2}(s, t) \leq L \int_{0}^{L} u_{x}^{2} \mathrm{~d} x, \quad \phi^{2}(s, t) \leq L \int_{0}^{L} \phi_{x}^{2} d x \quad s \in[0, L], \\
a b \leq \delta^{2} a^{2}+b^{2} / \delta^{2}, \quad a, b, \delta \in \mathbf{R}, \quad \delta \neq 0,
\end{gathered}
$$

where (17) follows from boundary conditions and Jensen's inequality, see Royden (1968),

Lemma 1. The operator $A$ given by (14) generates a $C_{0}$-semigroup in $\mathscr{H}$, (for the terminology on semigroup theory, the reader is referred to, e.g. Pazy (1983)).

Proof. We write $A=A_{U}+A_{\mathrm{B}}$ where,

$$
A_{U} y=\left[\begin{array}{c}
u_{2} \\
\frac{K}{\rho} \frac{\partial^{2} u_{1}}{\partial x^{2}} \\
\phi_{2} \\
\frac{E I}{I_{\rho}} \frac{\partial^{2} \phi_{1}}{\partial x^{2}} \\
A_{1} x_{1}+b_{1} u_{2}(L) \\
A_{2} x_{2}+b_{2} \phi_{2}(L)
\end{array}\right]
$$

where $D\left(A_{\mathrm{U}}\right)=D(A)$ and $y=\left(u_{1} u_{2} \phi_{1} \phi_{2} x_{1} x_{2}\right)^{T} \in \mathscr{H}$.

Using (17) and (18), it can be shown that $A_{\mathrm{B}}$ is a bounded linear operator on $\mathscr{H}$. Therefore, it is enough to show that $A_{U}$ generates a $C_{0}$-semigroup on $\mathscr{H}$, see Pazy (1983). To show the latter, we use the Lumer-Phillips theorem. Hence, we have to show that for some $c>0$, we have:

$$
\left\langle A_{\mathrm{U}} y, y\right\rangle_{\mathscr{H}} \leq c\|y\|_{\mathscr{H}}^{2}, \quad y \in D(A),
$$

where $\|\cdot\|_{\mathscr{F}}^{2}$ is the norm induced by (16), and for sufficiently large $\lambda, \lambda>c$, we have Range of $\left(\lambda I-A_{\mathrm{U}}\right)=\mathscr{H}$ where $I$ is the identity operator on $\mathscr{H}$.

Integrating by parts and using the boundary conditions, we obtain:

$$
\begin{aligned}
\left\langle A_{U} y, y\right\rangle_{\mathscr{X}}= & K u_{2}(L) u_{1 x}(L)+E I \phi_{2}(L) \phi_{1 x}(L) \\
& +x_{1}^{T}\left(A_{1}^{T} P_{1}+P_{1} A_{1}\right) x_{1} \\
& +x_{2}^{T}\left(A_{2}^{T} P_{2}+P_{2} A_{2}\right) x_{2}+2 x_{1}^{T} P_{1} b_{1} u_{2}(L) \\
& +2 x_{2}^{T} P_{2} b_{2} \phi_{2}(L) \leq\left[K \delta^{2}-\gamma_{1}\right] u_{2}^{2}(L) \\
& +\frac{L}{\delta^{2}} \int_{0}^{L} \phi_{x}^{2} d x \leq \frac{L}{\delta^{2} E I}\|z\|_{\mathscr{X}}^{2},
\end{aligned}
$$

where a first subscript denotes a component of a vector and the second subscript denotes a partial differential, i.e. $u_{1 x}=$ $\frac{\partial u_{1}}{\partial x}$, and $\delta \neq 0$ is chosen as $K \delta^{2}-\gamma_{1}<0$. To obtain the first equation, we used integration by parts and (4), (5), (6) and (9), and to obtain the first inequality, we used (17) and (18).

To prove that the Range of $\left(\lambda I-A_{U}\right)=\mathscr{H}$, we show that for any $\lambda>0$ and for any $\left(f_{1} f_{2} g_{1} g_{2} x_{1} x_{2}\right)^{T} \in \mathscr{H}$, we can find a $\left(u_{1} u_{2} \phi_{1} \phi_{2} z_{1} z_{2}\right)^{T} \in D(A)$ such that:

$$
\begin{gathered}
\lambda u_{1}-u_{2}=f_{1}, \quad \lambda u_{2}-\frac{K}{\rho} u_{1 x x}=f_{2}, \\
\lambda \phi_{1}-\phi_{2}=g_{1}, \quad \lambda \phi_{2}-\frac{E I}{I_{\rho}} \phi_{1 x x}=g_{2}, \\
\left(\lambda I-A_{1}\right) z_{1}-b_{1} u_{2}(L)=x_{1}, \\
\left(\lambda I-A_{2}\right) z_{2}-b_{2} \phi_{2}(L)=x_{2} .
\end{gathered}
$$


Using the techniques presented in Kim and Renardy (1987), it can easily be shown that (22)-(24) admits a solution in $D(A)$. In fact, $u_{1}$ and $\phi_{1}$ are given by:

$$
\begin{array}{r}
u_{1}(x)=K_{1} \sinh \mu_{1} x-\frac{\rho}{\mu_{1} K} \int_{0}^{x}\left[f_{2}(s)\right. \\
\left.+\lambda f_{1}(s)\right] \sinh \mu_{1}(x-s) \mathrm{d} s, \\
\begin{aligned}
\phi_{1}(x)=K_{2} \sinh \mu_{2} x-\frac{I_{\rho}}{\mu_{2} E I} \int_{0}^{x} & {\left[g_{2}(s)\right.} \\
& \left.+\lambda g_{1}(s)\right] \sinh \mu_{2}(x-s) \mathrm{d} s,
\end{aligned}
\end{array}
$$

where $K_{1}$ and $K_{2}$ are constant real numbers, $\mu_{1}=\lambda \sqrt{\rho / K}$, $\mu_{2}=\lambda \sqrt{I_{\rho} / K}$. Then, $u_{2}$ and $\phi_{2}$ are determined from (22) and (23), respectively, and the constants $K_{1}$ and $K_{2}$ are uniquely determined from $K\left(\phi_{1}(L)-u_{1 x}(L)\right)-c_{1}^{T} z_{1}-d_{1} u_{2}(L)=0$, $E I \phi_{1 x}(L)+c_{2}^{T} z_{2}+d_{2} \phi_{2}(L)=0$

3. Exponential decay of the solutions

Let $E_{\mathrm{B}}(t)$ be given by (3). We first define the following natural "energy" of the system:

$$
E(t)=E_{\mathrm{B}}(t)+w_{1}^{T}(t) P_{1} w_{1}(t)+w_{2}^{T}(t) P_{2} w_{2}(t) .
$$

It can easily be shown that for some constants $m_{1}>0$, $m_{2}>0$, the following holds:

$$
m_{1}\|z\|_{\mathscr{X}}^{2} \leq E(t) \leq m_{2}\|z\|_{\mathscr{X}}^{2} \text {. }
$$

Lemma 2. The energy $E(t)$ given by (27) is a nonincreasing function of time along the classical solutions of (13).

Proof. Differentiating (27) with respect to time, and using (13), we obtain:

$$
\begin{aligned}
\mathrm{d} E(t) / \mathrm{d} t & =-\gamma_{1} u_{t}^{2}(L, t)-\gamma_{2} \phi_{t}^{2}(L, t) \\
- & {\left[\sqrt{d_{1}-\gamma_{1}} u_{t}(L, t)-w_{1}^{T}(t) q_{1}\right]^{2}-\left[\sqrt{d_{2}-\gamma_{2}} \phi_{t}(L, t)\right.} \\
& \left.-w_{2}^{T}(t) q_{2}\right]^{2}-\varepsilon_{1} w_{1}^{T}(t) Q_{1} w_{1}(t)-\varepsilon_{2} w_{2}^{T}(t) Q_{2} w_{2}(t),
\end{aligned}
$$

where to obtain (29), we differentiated (27) with respect to time, and used (1), (2), (6), then we used integration by parts, (4)-(6), and (9). Since $d E(t) / d t$ is nonpositive, it follows that $E(t)$ is a nonincreasing function of time along the classical solutions of (13). $\quad \square$

Next we state and prove our main result:

Theorem 1. Let $T(t)$ be the $C_{0}$-semigroup in $\mathscr{H}$ generated by $A$ defined in (14). Then, the operator norm of $T(t)$ satisfies:

$$
\|T(t)\|^{2} \leq M \mathrm{e}^{-\sigma t}, \quad t \geq 0,
$$

for some positive constants $M$ and $\sigma$.

Proof. As in Kim and Renardy (1987) and Chen (1987), we first define the following function $V(t)$ :

$$
\begin{aligned}
V(t)=2(1-\varepsilon) t E(t)+2 \rho \int_{0}^{L} x u_{t} u_{x} \mathrm{~d} x & \\
+2 I_{\rho} \int_{0}^{L} x \phi_{x} \phi_{t} \mathrm{~d} x+\delta I_{\rho} \int_{0}^{L} \phi \phi_{t} \mathrm{~d} x & -\delta \rho \int_{0}^{L} u u_{t} \mathrm{~d} x,
\end{aligned}
$$

where $\varepsilon \in(0,1)$ and $\delta \in(0,1)$ are constants to be determined later. To prove the assertion, we first show that for a constant $C>0$, the following holds:

$[2(1-\varepsilon) t-C] E(t) \leq V(t) \leq[2(1-\varepsilon) t+C] E(t), \quad t \geq 0$.

Then we show that there exists a $T \geq 0$ such that:

$$
\frac{\mathrm{d} V(t)}{\mathrm{d} t} \leq 0, \quad t \geq T
$$

From (28), (29), (32) and (33) it follows that

$$
\int_{0}^{\infty}\|z\|_{\mathscr{X}}^{4} \mathrm{~d} t<\infty
$$

hence, (30) follows from a result due to Pazy (1983).
To prove (32), we first define the following quantities:

$$
\begin{aligned}
& I_{1}:=2 \rho \int_{0}^{L} x u_{t} u_{x} \mathrm{~d} x, \quad I_{2}:=2 I_{\rho} \int_{0}^{L} x \phi_{x} \phi_{t} \mathrm{~d} x, \\
& I_{3}:=\delta I_{\rho} \int_{0}^{L} \phi \phi_{t} \mathrm{~d} x, \quad I_{4}:=-\delta \rho \int_{0}^{L} u u_{t} \mathrm{~d} x .
\end{aligned}
$$

Using (16), (17) and (28), we obtain the following estimates:

$$
\begin{array}{r}
\left|I_{1}\right| \leq 2 \rho L\left(\int_{0}^{L} u_{x}^{2} \mathrm{~d} x+\int_{0}^{L} u_{t}^{2} \mathrm{~d} x\right) \leq L_{1} E(t), \\
\left|I_{2}\right| \leq 2 I_{\rho} L\left(\int_{0}^{L} \phi_{x}^{2} \mathrm{~d} x+\int_{0}^{L} \phi_{t}^{2} \mathrm{~d} x\right) \leq L_{2} E(t), \\
\left|I_{3}\right| \leq \delta I_{\rho}\left(\int_{0}^{L} \phi^{2} \mathrm{~d} x+\int_{0}^{L} \phi_{t}^{2} \mathrm{~d} x\right) \\
\leq \delta I_{\rho}\left(L^{2} \int_{0}^{L} \phi_{x}^{2} \mathrm{~d} x+\int_{0}^{L} \phi_{t}^{2} \mathrm{~d} x\right) \leq L_{3} E(t), \\
\left|I_{4}\right| \leq \delta \rho\left(\int_{0}^{L} u^{2} \mathrm{~d} x+\int_{0}^{L} u_{t}^{2} \mathrm{~d} x\right) \\
\leq \delta \rho\left(L^{2} \int_{0}^{L} u_{x}^{2} \mathrm{~d} x+\int_{0}^{L} u_{t}^{2} \mathrm{~d} x\right) \leq L_{4} E(t),
\end{array}
$$

where $L_{1}, L_{2}, L_{3}, L_{4}$ are some positive constants. Using (37)-(40) in (31), we obtain (32) with $C=L_{1}+L_{2}+L_{3}+$ $L_{4}$.

Using integration by parts, (1)-(3), we obtain:

$$
\begin{aligned}
\frac{\mathrm{d} I_{1}}{\mathrm{~d} t}=\rho L u_{f}^{2}(L, t)+K L & u_{x}^{2}(L, t)-\rho \int_{0}^{L} u_{t}^{2} \mathrm{~d} x \\
& -K \int_{0}^{L} u_{x}^{2} \mathrm{~d} x-2 K \int_{0}^{L} x \phi_{x} u_{x} \mathrm{~d} x
\end{aligned}
$$

$\frac{\mathrm{d} I_{2}}{\mathrm{~d} t}=I_{\rho} L \phi_{t}^{2}(L, t)-I_{\rho} \int_{0}^{L} \phi_{t}^{2} \mathrm{~d} x$

$$
\begin{gathered}
+E I L \phi_{x}^{2}(L, t)-E I \int_{0}^{L} \phi_{x}^{2} \mathrm{~d} x \\
+2 K \int_{0}^{L} x \phi_{x} u_{x} \mathrm{~d} x-K L \phi^{2}(L, t)+K \int_{0}^{L} \phi^{2} \mathrm{~d} x,
\end{gathered}
$$

$\frac{\mathrm{d} I_{3}}{\mathrm{~d} t}=\delta I_{\rho} \int_{0}^{L} \phi_{t}^{2} \mathrm{~d} x+\delta E I \phi(L, t) \phi_{x}(L, t)$

$$
\begin{aligned}
-\delta E I \int_{0}^{L} \phi_{x}^{2} \mathrm{~d} x+\delta K \phi(L, t) u(L, t) \\
-\delta K \int_{0}^{L} u \phi_{x} \mathrm{~d} x-\delta K \int_{0}^{K} \phi^{2} \mathrm{~d} x
\end{aligned}
$$

$\frac{\mathrm{d} I_{4}}{\mathrm{~d} t}=-\delta \rho \int_{0}^{L} u_{t}^{2} \mathrm{~d} x-\delta K u(L, t) u_{x}(L, t)$

$$
+\delta K \int u_{x}^{2} \mathrm{~d} x+\delta K \int u \phi_{x} \mathrm{~d} x
$$

Using (5), (6), (17) and (18), we obtain the following estimates:

$$
\int_{0}^{L}\left(u_{x}-\phi\right)^{2} \mathrm{~d} x \leq 2 \int_{0}^{L} u_{x}^{2} \mathrm{~d} x+2 L^{2} \int_{0}^{L} \phi_{x}^{2} \mathrm{~d} x,
$$

$K L u_{x}^{2}(L, t) \leq K L \phi^{2}(L, t)$

$$
+(4 L / K)\left(c_{1}^{T} w_{1}(t)\right)^{2}+\left(4 L d_{1}^{2} / K\right) u_{f}^{2}(L, t)
$$

$E I L \phi_{x}^{2}(L, t) \leq(2 L / E I)\left(c_{2}^{T} w_{2}(t)\right)^{2}+\left(2 L d_{2}^{2} / E I\right) \phi_{t}^{2}(L, t)$,

$\delta E I \phi(L, t) \phi_{x}(L, t) \leq \delta \delta_{1}^{2} L \int_{0}^{L} \phi_{x}^{2} \mathrm{~d} x$

$$
+\left(2 \delta / \delta_{1}^{2}\right)\left(c_{2}^{T} w_{2}(t)\right)^{2}+\left(2 \delta d_{2}^{2} / \delta_{1}^{2}\right) \phi_{1}^{2}(L, t),
$$

$\delta K\left[\phi(L, t)-u_{x}(L, t)\right] u(L, t) \leq \delta \delta_{2}^{2} L \int_{0}^{L} u_{x}^{2} \mathrm{~d} x$

$$
+\left(2 \delta / \delta_{2}^{2}\right)\left(c_{1}^{T} w_{1}(t)\right)^{2}+\left(2 \delta d_{1}^{2} / \delta_{2}^{2}\right) u_{t}^{2}(L, t),
$$


where $\delta_{1} \neq 0, \delta_{2} \neq 0$ are arbitrary constants. Differentiating (31) with respect to time, using (29), (41)-(49), and collecting likewise terms, we obtain:

$$
\begin{aligned}
\frac{\mathrm{d} V(t)}{\mathrm{d} t} \leq & -\left[2(1-\varepsilon) t \varepsilon_{1} w_{1}^{T}(t) Q_{1} w_{1}(t)-2(1\right. \\
& -\varepsilon) w_{1}^{T}(t) P_{1} w_{1}(t)-((4 L / K) \\
& \left.\left.+\left(2 \delta / \delta_{2}^{2}\right)\right)\left(c_{1}^{T} w_{1}(t)\right)^{2}\right] \\
& -\left[2(1-\varepsilon) t \varepsilon_{2} w_{2}^{T}(t) Q_{2} w_{2}(t)\right. \\
& -2(1-\varepsilon) w_{2}^{T}(t) P_{2} w_{2}(t)-((2 L / E I) \\
& \left.\left.+\left(2 \delta / \delta_{1}^{2}\right)\right)\left(c_{2}^{T} w_{2}(t)\right)^{2}\right] \\
& -(\varepsilon+\delta) \rho \int_{0}^{I} u_{t}^{2} \mathrm{~d} x-[(\varepsilon+\delta) E I \\
& \left.+(2 \varepsilon+\delta-3) K L^{2}-\delta \delta_{1}^{2} L\right] \int_{0}^{L} \phi_{x}^{2} \mathrm{~d} x \\
& -(\varepsilon-\delta) I_{\rho} \int_{1}^{L} \phi_{t}^{2} \mathrm{~d} x-[(2 \varepsilon-\delta-1) K \\
& \left.-\delta \delta_{2}^{2} L\right] \int_{0}^{L} u_{x}^{2} \mathrm{~d} x \\
& -2(1-\varepsilon) t\left[\sqrt{d_{1}-\gamma_{1}} u_{t}(L, t)-w_{1}^{T}(t) q_{1}\right]^{2} \\
& -2(1-\varepsilon) t\left[\sqrt{d_{2}-\gamma_{2}} \phi_{t}(L, t)-w_{2}^{T}(t) q_{2}\right]^{2} \\
& -\left[2(1-\varepsilon) t \gamma_{1}-\rho L-\left(4 L d_{1}^{2} / K\right)\right. \\
& \left.-\left(2 \delta d_{1}^{2} / \delta_{2}^{2}\right)\right] u_{t}^{2}(L, t) \\
& -\left[2(1-\varepsilon) t \gamma_{2}-I_{\rho} L-\left(2 L d_{2}^{2} / E I\right)\right. \\
& \left.-\left(2 \delta d_{2}^{2} / \delta_{1}^{2}\right)\right] \phi_{t}^{2}(L, t) .
\end{aligned}
$$

Let us choose $\varepsilon, \delta, \in[0,1]$, and $\delta_{1} \neq 0, \delta_{2} \neq 0$ sufficiently small such that:

$$
\begin{gathered}
\frac{\delta}{1-\delta}>\frac{K L^{2}}{E I}, \quad \varepsilon-\delta>0, \\
(2 \varepsilon-\delta-1) K-\delta \delta_{2}^{2} L>0, \\
2 \varepsilon-\delta-1>0, \\
(\varepsilon+\delta) E I+(2 \varepsilon+\delta-3) K L^{2}-\delta \delta_{1}^{2} L>0 .
\end{gathered}
$$

The above inequalities always have a solution, for example by choosing $\delta=\left(E I+2 K L^{2}\right) /\left(2 E I+2 K L^{2}\right), \quad \varepsilon=(7 E I+$ $\left.8 K L^{2}\right) /\left(8 E I+8 K L^{2}\right)$, and then $\delta_{1}^{2}<E I\left(11 E I+10 K L^{2}\right) /$ $\left(8 \delta L E I+8 \delta K L^{3}\right)$ and $\delta_{2}^{2}<E I K /\left(4 \delta L E I+4 \delta K L^{3}\right)$, we see that the above inequalities are satisfied. Then, from $(50)$ it follows that there exists a $T \geq 0$ depending only on $\varepsilon, \delta, \delta_{1}$, $\delta_{2}, \varepsilon_{1}, \varepsilon_{2}$ and the coefficients in (1), (2), such that (33) holds. Therefore, using the argument following (33), we arrive at $(30)$.

Remark 1. In case of a non-dynamic feedback, (6) reduces to:

$$
f_{1}(t)=d_{1} u_{t}(L, t), \quad f_{2}(t)=d_{2} \phi_{1}(L, t)
$$

which is the case considered in Kim and Renardy (1987).

Remark 2. We note that both of the actuator transfer functions given by the control laws (6) and (51) are not band-limited, see (8). Also, the restriction of actuator transfer functions to positive real functions might seem to be too restrictive, but nevertheless this type of actuator transfer functions are more general than those given by (51). Moreover, the proposed control laws are more suitable for some control applications, such as eigenvalue assignment, disturbance rejection, etc. than the control law given by (51). This point will be explained below and in Section 4

One way of implementing the control laws given by $(51)$ is to use actuators whose inputs are $u_{t}(L, t)$ and $\phi_{t}(L, t)$, and whose outputs are $f_{1}(t)$ and $f_{2}(t)$, where the actuator transfer functions are given by $d_{1}$ and $d_{2}$, respectively. From a practical point of view, however, most actuators show some dynamic behaviour, at least over a frequency range, hence their transfer functions are not constants. In this case,
Theorem 1 provides a sufficient condition to ensure exponential stability, whereas the results of $\mathrm{Kim}$ and Renardy (1987) do not apply.

Also note that the proposed dynamic control, (6), as well as the standard non-dynamic one, (51), change the frequency-domain characteristic of the uncontrolled system; that is the eigenvalues of the operator given by (14) are completely different from the eigenvalues of the uncontrolled system. This change in the spectrum, although limited, may possibly be used for some control applications, such as eigenvalue assignment, disturbance rejection, etc. Note that the dynamic control offers more degrees of freedom to change the spectrum of the operator given by (14), than the standard non-dynamic one. For example, simulation studies of Chen (1987) and Kim and Renardy, (1987), show that the non-dynamic boundary control affects the eigenvalue of the system uniformly, whereas by using dynamic boundary control, it may be possible to change the spectrum of the system only over a prescribed frequency range, without changing the rest of the spectrum very much. In the next section, we give some simulation results indicating this use of dynamic boundary control.

\section{Numerical results}

In this section, to show the effect of the proposed control laws given by (6), on the eigenvalues of the system given by (14), we present some numerical simulation results. We use normal mode analysis and set:

$$
\begin{aligned}
u(x, t) & =\mathrm{e}^{\lambda t} U(x), \\
\phi(x, t) & =\mathrm{e}^{\lambda t} \Phi(x), \\
w_{i}(t) & =\mathrm{e}^{\lambda t} W_{i}, i=1,2,
\end{aligned}
$$

where $x \in[0, L], t \geq 0, U, \Phi:[0, L]: \rightarrow \mathbf{R}$ are appropriate functions, $W_{i} \in \mathbf{R}^{n_{i}}, i=1,2$ are appropriate constant vectors and $\lambda$ is the eigenvalue to be determined. To find the eigenvalues satisfying (52) and (1), (2), (4)-(6), we use finite difference technique with $N$ point spatial discretization, approximating the spatial derivatives by using a central difference formula, see Greenspan and Casulli (1988). The resulting equations can be rewritten in the form $\operatorname{det}\left(\lambda^{2} P+\right.$ $\lambda Q+R)=0$ where $P, Q, R \in \mathbf{R}^{m \times m}, m=2 N+n_{1}+n_{2}$, are appropriate matrices. This equation takes on the customary form: $A=\lambda B$ where $A, B \in \mathbf{R}^{2 m \times 2 m}$ are given as:

$$
A=\left[\begin{array}{cc}
0 & -R \\
I & Q
\end{array}\right], \quad B=\left[\begin{array}{cc}
-I & 0 \\
0 & -P
\end{array}\right]
$$

In the simulations we use the following set of parameters which model a solid aluminium bar: $\rho=40 \mathrm{~kg} \mathrm{~m}^{-1}, K=$ $2.8 \times 10^{8} \mathrm{kgm} \mathrm{sec}^{-2}, \quad E I=6.3 \times 10^{5} \mathrm{sec}^{-2}, \quad I_{\rho}=0.0332 \mathrm{kgm}$, $L=2 \mathrm{~m}$.

To see the difference between the effects of the non-dynamic and the dynamic boundary control on the eigenvalues we first consider the non-dynamic boundary control as given by (51) with the following parameters:

Case 1: $d_{1}=1, d_{2}=1$.

By comparing the results of $N=50$ and $N=55$, we conclude that about 27 complex conjugate pairs have converged at $N=50$, with relative error of both real and imaginary parts of the eigenvalues less than $1 \%$. Due to space limitation, we present only the first five lowest eigenvalues. Note that all converged eigenvalues have negative real parts which decrease (i.e. damping increases) as the imaginary parts increase, in accordance with the results of Kim and Renardy (1987).

For some control applications it may be desirable to change the spectrum only over a prescribed frequency range. For example, the beam may be subject to a disturbance with a known frequency context. In this case, to reduce the effect of the disturbance, it may be desirable to introduce more damping only to the modes of the beam over the frequency range of the disturbance.

From Table 1, we see that the higher modes are damped rather well, but the damping associated with the lower modes, especially the first and second modes, are rather small. Therefore we may want to introduce more damping to 
Table 1. Eigenvalues for Case 1

$1.0 \times 10^{5} \star$

$-0.00000043228545 \pm 0.00119485753223$ $-0.00000192237411 \pm 0.00740277945165 i$

$-0.00000449568932 \pm 0.02036214818773 i$

$-0.00000806812670 \pm 0.03892750846202 i$

$-0.00001229204976 \pm 0.06244736533499 i$

Table 2. Eigenyalues for Case 2

$1.0 \times 10^{5} \star$

$-0.00000516460452 \pm 0.00119485857851$

$-0.00000192796170 \pm 0.00740309743653 i$

$-0.00000449362672 \pm 0.02036241081321$

$-0.00000807053831 \pm 0.03892776239342$

$-0.00001228631474 \pm 0.06244758839125 i$

Table 3. Eigenvalues for Case 3

$1.0 \times 10^{5} \star$

$-0.00000086525939 \pm 0.00119485760277$

$-0.00000197409347 \pm 0.00740308902514 i$

$-0.00000450748096 \pm 0.02036241038715 i$

$-0.00000807875856 \pm 0.3892775964977 i$

$-0.00001228868545 \pm 0.06244759418429$

Table 4. Eigenvalues for Case 4

$1.0 \times 10^{5}$

$-0.00000087102608 \pm 0.00119485782482$

$-0.00000192340624 \pm 0.00740281189987 i$

$-0.000004492621268 \pm 0.02036217141781 i$

$-0.00000807099807 \pm 0.03892753940502 i$

$-0.00001228458524 \pm 0.06244738387793 i$

Table 5. Eigenvalues for Case 5

$1.0 \times 10^{5} \star$

$-0.00005969479095 \pm 0.00119290900150$

$-0.00000197299698 \pm 0.00740596154149 i$

$-0.00000450397047 \pm 0.02036479425832 i$

$-0.00000807341702 \pm 0.03892998790025 i$

$-0.0000122828955 \pm 0.06244970471820 i$

Table 6. Eigenvalues for Case 6

$1.0 \times 10^{5} \star$

$-0.00000043346442 \pm 0.00119478592758 i$

$-0.00002239037315 \pm 0.00740285578075 i$

$-0.00000456806342 \pm 0.02036401853819 i$

$-0.00000809900202 \pm 0.03892910667506 i$

$-0.00001229928068 \pm 0.06244883025543 i$

Table 7. Eigenvalues for CASE 7

$1.0 \times 10^{5} \star$

$-0.00000355607727 \pm 0.00119483237520$

$-0.00001914733864 \pm 0.00740290908041$

$-0.00000456294907 \pm 0.02036392203410 i$

$-0.00000809851705 \pm 0.03892905890156 i$

$-0.00001229725497 \pm 0.06244880700766 i$ the first two modes without changing much the remaining ones. Note that this could be achieved by increasing $d_{1}$ and $d_{2}$, but in that case the remaining modes are also affected uniformly (see Kim and Renardy (1987)), and the required actuator energy will possibly increase, which may cause saturation in the actuator.

To introduce more damping only to the lower modes, we propose the following actuator transfer function:

$$
h_{i}(s)=d_{i}+\frac{K_{i} s}{s^{2}+2 \xi_{i} \omega_{0 i} s+\omega_{0 i}^{2}}, \quad i=1,2,
$$

where, for $i=1,2, K_{i}, \xi_{i}$ and $\omega_{0 i}^{2}$ are positive constants.

The real part of $h_{i}(j \omega)$ is given by:

$$
\mathscr{R} e\left\{h_{i}(j \omega)\right\}=d_{i}+\frac{2 K_{i} \xi_{i} \omega_{0 i} \omega^{2}}{\left(\omega_{0 i}^{2}-\omega^{2}\right)^{2}+4 \xi_{i}^{2} \omega_{0 i}^{2} \omega^{2}}, \quad i=1,2,
$$

hence (8) is satisfied with $\gamma_{i}=d_{i}$, for $i=1,2$. The maximum of $\mathscr{R}\left\{h_{i}(j \omega)\right\}$ is obtained at $\omega=\omega_{0 i}$ and is given by:

$$
\max _{\omega \in \mathbb{R}} \Re_{e}\left\{h_{i}(j \omega)\right\}=d_{i}+\frac{K_{i}}{2 \xi_{i} \omega_{0 i}}, \quad i=1,2,
$$

and $\mathscr{R} e\left\{h_{i}(j \omega)\right\}$ decreases to $d_{i}$ as $\omega \rightarrow 0$ and $\omega \rightarrow \infty$. Also note that the proposed dynamic controller does not increase uniformly the minimum of $\mathscr{R}\left\{\left\{h_{i}(j \omega)\right\}\right.$, that is:

$$
\inf _{\omega \in \mathbf{R}} \mathscr{R} e\left\{h_{i}(j \omega)\right\}=d_{i}, \quad i=1,2 .
$$

Since we want to decrease $\mathscr{R} e\left\{\lambda_{1}\right\}$ and $\mathscr{R} e\left\{\lambda_{2}\right\}$, where $\lambda_{1}$ and $\lambda_{2}$ are first and second eigenvalues in Table 1 , respectively, from the reasoning above we conclude that a good choice for this purpose is $\omega_{0 i}=\mathscr{I}_{m}\left\{\lambda_{i}\right\}$ or $\omega_{0 i}=$ $\mathscr{I}_{m}\left\{\lambda_{2}\right\}$. For $N=50$, we calculated the eigenvalues for the following choices of the actuator parameters for $i=1,2$

Case 2: $d_{i}=1, K_{1}=119.48, \xi_{i}=0.05, \omega_{0 i}=119.48$,

Case 3: $d_{i}=1, K_{i}=119.48, \xi_{i}=0.5, \omega_{0 i}=119.48$,

Case 4: $d_{i}=1, K_{i}=11.948, \xi_{i}=0.05, \omega_{0 i}=119.48$,

Case 5: $d_{i}=1, K_{i}=1194.8, \xi_{i}=0.05, \omega_{0 i}=119.48$,

Case 6: $d_{i}=1, K_{i}=740.28, \xi_{i}=0.05, \omega_{0 i}=740.48$,

Case 7: $d_{i}=1, K_{1}=119.48, K_{2}=740.28, \xi_{i}=0.05, \omega_{01}=$ $119.48, \omega_{02}=740.28$.

In all cases, the five lowest eigenvalues associated with the beam vibrations are given in the Tables 2-7, respectively.

As it can be seen from Tables 2-6, the resonant frequency $\omega_{0 i}$ of the actuator determines the frequency of the mode which is affected by the control law; decreasing the damping constant $\xi_{i}$ and/or increasing the gain $K_{i}$ have increasing effect on the damping of the mode of interest, as expected from (56). Also from Table 7, we see that it may be possible to introduce more damping only to the lowest modes by using different actuators for force and moment control.

The results of these simulations suggest that it might be possible to change the spectrum of the system given by (1)-(5) over a specified frequency range, without altering the remaining part of the spectrum very much. This point, as well as possible applications of the ideas presented in this section, need further investigation.

\section{Conclusion}

In this paper we considered the stabilization of a clamped-free Timoshenko beam using dynamic boundary control. Under some assumptions, one of which is the positive realness of the actuator transfer functions corresponding to the dynamic boundary controls, we proved that the energy of the actuator-beam configuration decays exponentially to zero. We also give some numerical simulation results. These simulations results suggest that it might be possible to change the spectrum of the system over a specified frequency range by using dynamic boundary control without affecting the remaining part of the spectrum. Previous numerical simulation results suggest that one may not obtain such a result by using only non-dynamic feedback, see Chen (1987) and Kim and Renardy (1987). This change in the spectrum could be used in some control applications, such as eigenvalue assignment, disturbance rejection, etc. 


\section{References}

Chen, G. (1979). Energy decay estimates and exact boundary value controllability for the wave equation in a bounded domain. J. Math. Pures. Appl., 58, 249-273.

Chen, G. M., M. C. Delfour, A. M. Krall and G. Payre (1987). Modelling, stabilization and control of serially connected beams. SIAM J. of Contr. and Optim., 25, 526-546.

Greenspan, D. and V. Casulli (1988). Numerical Analysis for Applied Mathematics, Science and Engineering, AddisonWesley, Reading, MA

Kim, J. U. and Y. Renardy (1987). Boundary control of the Timoshenko beam. SIAM J. of Control and Optim., 25, 1417-1429.

Lagnese, J. E. (1989). Boundary stabilization of thin plates. SIAM Studies in Applied Mathematics, Vol. 10

Meirovitch, L. (1967). Analytical Methods in Vibrations.
MacMillan, New York.

Morgül, Ö. (1990). Control and stabilization of a flexible beam attached to a rigid body, Int. J. of Control, 51, $11-31$

Morgül, Ö. (1990). Control and stabilization of a flexible beam attached to a rigid body. Int. J. of Control, 51, $11-31$

Morgül, Ö. (1991a). Orientation and stabilization of a flexible beam attached to a rigid body: planar motion. IEEE Trans. on Aut. Control, 36, 953-963.

Pazy, A. (1983). Semigroups of Linear Operators and Applications to Partial Differential Equations. SpringerVerlag, New York

Royden, H. L. (1968). Real Analysis, 2nd ed MacMillan New York.

Vidyasagar, M. (1978). Nonlinear Systems Analysis. Englewood Cliffs, Prentice-Hall, NJ. 